Draft Version June 17, 2021

Preprint typeset using $\mathrm{L}^{A} \mathrm{~T}_{\mathrm{E}} \mathrm{X}$ style emulateapj v. 08/22/09

\title{
IGR J22517+2218=MG3 J225155+2217: A NEW GAMMA-RAY LIGHTHOUSE IN THE DISTANT UNIVERSE
}

\author{
L. Bassani ${ }^{1}$, R. Landi $^{1}$, A. Malizia ${ }^{1}$, M.T. Fiocchi ${ }^{2}$, A. Bazzano ${ }^{2}$, A. J. Bird ${ }^{3}$, A. J. Dean ${ }^{3}$, N. Gehrels $^{4}$, \\ P. Giommi ${ }^{4}$, P. Ubertini ${ }^{2}$ \\ Draft version June 17, 2021
}

\begin{abstract}
We report on the identification of a new soft gamma ray source, namely IGR J22517+2218, detected with IBIS/INTEGRAL. The source, which has an observed 20-100 keV flux of $\sim 4 \times 10^{-11} \mathrm{erg} \mathrm{cm}^{-2}$ $\mathrm{s}^{-1}$, is spatially coincident with MG3 J225155+2217, a quasar at $\mathrm{z}=3.668$. The Swift $/ \mathrm{XRT} 0.5-10 \mathrm{keV}$ continuum is flat $(\Gamma=1.5)$ with evidence for a spectral curvature below $1-2 \mathrm{keV}$ either due to intrinsic absorption $\left(\mathrm{N}_{H}=3 \pm 2 \times 10^{22} \mathrm{~cm}^{-2}\right)$ or to a change in slope $(\Delta \Gamma=0.5)$. X-ray observations indicate flux variability over a 6 days period which is further supported by a flux mismatch between Swift and INTEGRAL spectra. IGR J22517+2218 is radio loud and has a flat radio spectrum; optically it is a broad line emitting quasar with the atypical property of hosting a narrow line absorption system. The Source Spectral Energy Distribution is unusual compared to blazars of similar type: either it has the synchrotron peak in the X/gamma-ray band (i.e. much higher than generally observed) or the Compton peak in the $\mathrm{MeV}$ range (i.e. lower than typically measured). IGR J22517+2218=MG3 $\mathrm{J} 225155+2217$ is the second most distant blazar detected above $20 \mathrm{keV}$ and a gamma-ray lighthouse shining from the edge of our Universe.

Subject headings: X-gamma-ray data- active galactic nuclei
\end{abstract}

\section{INTRODUCTION}

Blazars are the most powerful of all AGN seen in the observable Universe; their continuously emitting radiation covers the entire electromagnetic spectrum from radio to gamma-ray frequencies. Because of their enormous luminosities, blazars are visible to very large distances/redshifts. In the widely adopted scenario of blazars, a single population of high-energy electrons in a relativistic jet radiate from the radio/FIR to the UVsoft X-rays by the synchrotron process and at higher frequencies by inverse Compton scattering soft-target photons present either in the jet, in the surrounding material or in both (Ghisellini et al. 1998). Therefore a strong signature of the blazar nature of a source is a double peaked structure in the Spectral Energy Distribution (SED), with the synchrotron component peaking anywhere from infrared to X-rays and the inverse Compton extending up to $\mathrm{GeV} / \mathrm{TeV}$ gamma-rays. To explain all the different SEDs observed in blazars, Fossati et al. (1998) proposed the blazar's sequence, which claims an inverse relation between peak energies and source luminosity: the more luminous sources have both synchrotron and Inverse Compton peaks at lower energies than their fainter (and generally at lower redshift) counterparts. Within the blazars population, high redshift objects, which belong to the class of flat spectrum radio Quasars (FSRQ), tend to be the most luminous one. Their SED peak at IR/optical (first peak) and GeV (second peak) frequencies and only a handful of objects show both peaks at lower energies (Blom et al. 1996).

\footnotetext{
${ }^{1}$ IASF-Bologna/INAF, Via Gobetti 101, I-40129 Bologna, Italy

${ }^{2}$ IASF-Roma/INAF, Via Fosso del Cavaliere 100, I-00133 Rome, Italy

3 School of Physics and Astronomy, University of Southampton, Highfield, Southampton, SO 17 1BJ, UK.

4 NASA/Goddard Space Flight Center, Greenbelt, MD 20771

${ }^{5}$ ASI Science Data Center, ESRIN, I-00044 Frascati, Italy
}

Furthermore, FSRQ with a synchrotron peak above the optical band are extremely rare (Giommi et al. 2007) and not predicted by the blazar's sequence. In less luminous blazars, on the other hand, the first peak can reach the $\sim 100 \mathrm{keV}$ band while the second one goes well into the $\mathrm{TeV}$ range. Observations above $10 \mathrm{keV}$ are therefore extremely important as they can select rare type of blazars, i.e. those with the synchrotron or Inverse Compton peak at hard X-ray/ gamma-ray energies. Finding either type of objects is crucial to test the validity of the blazar's sequence and to study jet parameters in extreme blazars or, in other words, to stretch blazar theories to the limit. Unfortunately, observational progress in Blazar's studies above $10 \mathrm{keV}$ has been slow, particularly for the most distant sources. Nonetheless, a few objects at high $\mathrm{z}$ have been detected by BeppoSAX/PDS first (less than 10 in the 1-4.7 z range, Donato et al. 2005) and more recently by INTEGRAL/IBIS (Bird et al. 2007) and Swift/BAT (Sambruna et al. 2007). Here, we provide evidence for the identification of a newly discovered INTEGRAL/IBIS source, IGR J22517+2218, with a quasar (QSO) at $\mathrm{z}=3.668$ by means of follow up observations with Swift/XRT; we also present the combined $\mathrm{X} /$ gamma-ray spectrum of the source and discuss its peculiar SED. This is the second most distant blazar ever detected above $20 \mathrm{keV}$.

\section{IGR J22517+2218}

IGR J22517+2218 was first reported by Krivonos et al. (2007) as an unidentified object detected during revolution 316 (150 ksec exposure). Here, we have combined slightly more data to reach a total exposure of $190 \mathrm{ks}$. IBIS data have been reduced following the same procedure used for our survey work (Bird et al. 2007). The source is detected with a significance of $\sim 7 \sigma$ at R.A. $(2000)=22 \mathrm{~h} 51 \mathrm{~m} 42.72 \mathrm{~s}$ and $\operatorname{Dec}(2000)=+22^{\circ} 17^{\prime} 56.4^{\prime \prime}$ with a positional uncertainty of 4.5 ' (90\% confidence level see Figure.1); this position 


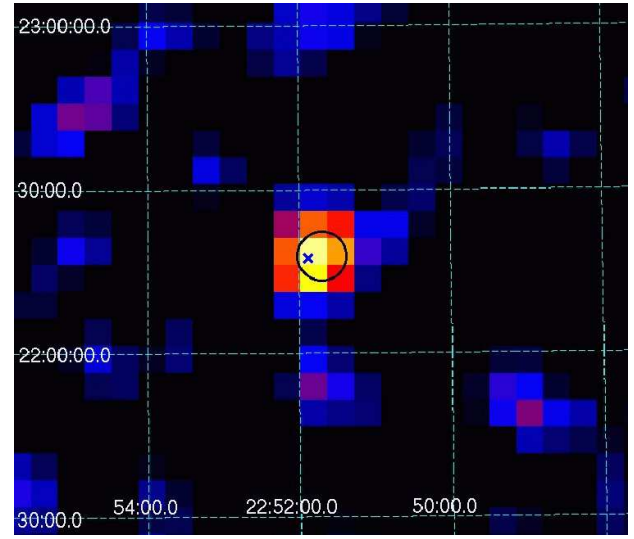

FIG. 1.- IBIS/ISGRI 20-100 keV image of IGR J22517+2218. The circle corresponds to the INTEGRAL/IBIS error box while the X is the optical position of MG3 J225155+2217.

is $32^{\circ}$ above the galactic plane, suggestive of an extragalactic nature. Our position is compatible with that reported by Krivonos et al (2007) (note that their 2.1' error radius corresponds to $68 \%$ confidence level). The source count rate in the $20-100 \mathrm{keV}$ band is $0.4 \pm 0.06$ counts $\mathrm{s}^{-1}$ (or $2.17 \pm 0.33 \mathrm{mCrab}$ ). We have divided all available observations in 3 sets of data to find evidence of flux variability but found none. The IBIS spectrum was extracted using the standard Off-line Scientific Analysis (OSA version 5.1) software released by the Integral Scientific Data Centre. Here and in the following, spectral analysis was performed with XSPEC v.11.3.2 package and errors are quoted at $90 \%$ confidence level for one interesting parameter $\left(\Delta \chi^{2}=2.71\right)$. A simple power law provides a good fit to the IBIS data $\left(\chi^{2}=6.5\right.$ for 8 d.o.f. $)$ and a photon index $\Gamma=1.4 \pm 0.6$ combined to an observed $20-100 \mathrm{keV}$ flux of $4 \times 10^{-11} \mathrm{erg} \mathrm{cm}^{-2} \mathrm{~s}^{-1}$. Within the INTEGRAL/IBIS error circle, we find the QSO MG3 $\mathrm{J} 225155+2217$ at $\mathrm{z}=3.668$; however its association with IGR J22517+2218 is not straightforward as 1) there are other potential candidates in the high energy error box for example at radio frequencies and 2) it is surprising to detect such a distant object in a short exposure with INTEGRAL. For this reason we have requested and obtained follow up observations of this sky region with the XRT telescope (Hill et al. 2004) on board the Swift satellite (Gehrels et al.2004).

\section{SWIFT/XRT FOLLOW UP OBSERVATION}

On May 21, 22 and 26, 2007 Swift/XRT carried out observations of IGR J225155+2217 lasting 1.6, 12.5 and $11.3 \mathrm{ks}$ respectively. Data were collected in Photon Counting mode and reduction was performed using the XRTDAS v2.0.1 standard data pipeline package. Events for spectral analysis were extracted within a circular region of radius $20 "$ (which encloses about $90 \%$ of the PSF at $1.5 \mathrm{keV}$, Moretti et al. 2004) centered on the source position. The background was taken from various source-free regions close to the X-ray source using circular/annular regions with different radii, in order to ensure an evenly sampled background. In all cases, the spectra were extracted from the corresponding event files using XSELECT software and binned using GRPPHA in an appropriate way, so that the $\chi^{2}$ statistic could reliably be used. We used the latest version (v.008) of the response matrices and create individual ancillary response

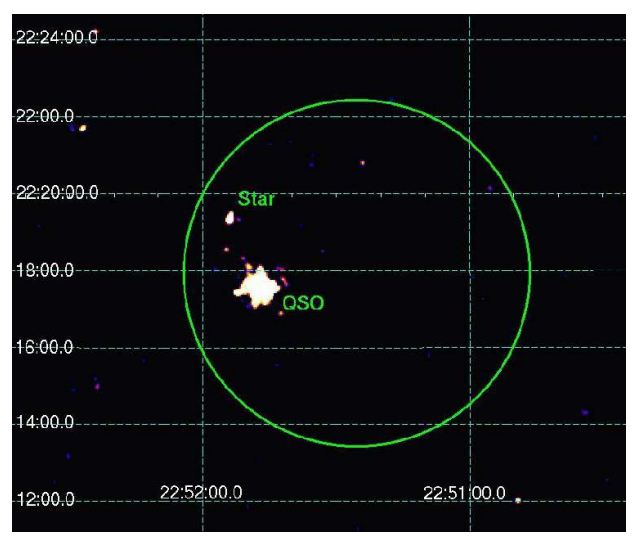

FIG. 2.- Swift/XRT (2-10 keV) image of IGR J225155+2217. The circle corresponds to the INTEGRAL/IBIS error box.

files (ARF) using XRTMKARF. In all our fitting procedures we have used a Galactic column density which in the direction of IGR J225155+2217 is $5 \times 10^{20} \mathrm{~cm}^{-2}$ (Dickey \& Lockman, 1990). Using the XIMAGE detection algorithm, we searched the XRT images $(0.3-10 \mathrm{keV}$ band) for significant excesses (above 3 sigma level) falling within the INTEGRAL/IBIS $90 \%$ confidence circle (figure 2); only one source (N1) was clearly detected in all 3 observations (at 10, 27 and $20 \sigma$ ) while a second one (N2) was seen (at $4.5 \sigma$ ) only in the longest XRT exposure. None of the other potential candidates in the IBIS error box is detected in X-rays. Table 1 reports for source N1 and N2 the XRT position, associated error radius and, for each observation, net count rates and fluxes. Source N1 is the high redshift QSO MG3 J225155+2217. Its XRT spectrum is hard and bright: a simple power law fit to the two longest observations provides $\Gamma=1.41 \pm 0.12$ $\left(\chi^{2}=28.9,17\right)$ and $\Gamma=1.30 \pm 0.08\left(\chi^{2}=34.4,41\right.$ dof $)$ together with a $2-10 \mathrm{keV}$ flux in the range $2.6-3.4 \times 10^{-12}$ erg $\mathrm{cm}^{-2} \mathrm{~s}^{-1}$. The XRT spectrum of source N2 is, instead, very soft as no emission is detected above $3 \mathrm{keV}$; it is well fitted by a thermal bremstrahulung model with $\mathrm{kT}=0.4_{-0.3}^{+0.1}\left(\chi^{2}=4,3 \mathrm{dof}\right)$ and a $2-10 \mathrm{keV}$ flux of $\sim 10^{-15}$ erg $\mathrm{cm}^{-2} \mathrm{~s}^{-1}$. Its positional coincidence with either a double (CCDM J22519+2219AB) or a single (TYC 1710969-1) star, suggests that the X-ray emission comes from a stellar corona; the late spectral type (F5) of CCDM $\mathrm{J} 22519+2219 \mathrm{AB}$ is fully compatible with this hypothesis. In any case, the much lower flux (more than 3 orders of magnitudes) and softer spectrum of source N2 compared to source N1 indicates that its emission is unlikely to extend into the INTEGRAL energy range and strongly argues in favour of the association of IGR J225155+2217 with the only other detected X-ray counterpart, i.e. the high z QSO.

To better characterized the QSO X-ray spectrum and in view of the fact that the INTEGRAL detection is over a few revolutions, i.e provides an average flux, we have combined the two most statistically significant XRT spectra and repeated the spectral analysis. To account for the high $\mathrm{z}$ value, all modeling has been carried out in the source rest frame. A simple power law fit to this stacked spectrum still provides a flat photon index $(\Gamma=1.31 \pm 0.09)$ and an acceptable $\chi^{2}(46.9 / 53)$. However, inspection of the residuals to this model reveals some curvature possibly due to intrinsic absorption in 
TABLE 1

Swift/XRT DETECTIONS

\begin{tabular}{|c|c|c|c|c|c|c|}
\hline Source & $\mathrm{RA}^{\dagger}$ & $\operatorname{Dec}^{\dagger}$ & error $^{\dagger}$ & CR/ Flux(obs1) ${ }^{\ddagger}$ & CR/ Flux $(\text { obs } 2)^{\ddagger}$ & CR/ Flux $(\text { obs } 3)^{\ddagger}$ \\
\hline N1 & 225153.44 & +221735.86 & 3.6 & $8.77 \pm 0.85 / 4.4 \pm 0.4$ & $8.36 \pm 0.31 / 3.4 \pm 0.1$ & $7.05 \pm 0.35 / 2.6 \pm 0.1$ \\
\hline $\mathrm{N} 2$ & 225156.70 & +221922.90 & 4.6 & $0.59 \pm 0.22 / \leq 0.003$ & $0.21 \pm 0.06 / 0.001 \pm 0.0002$ & $0.18 \pm 0.06 / \leq 0.001$ \\
\hline
\end{tabular}

Notes: ${ }^{\dagger}$ Positions and relative uncertainties (in arcsec) are calculated using the xrtcentroid (v0.2.7) task. $\ddagger$ Count rates (CR) are extracted from $0.3-10 \mathrm{keV}$ band image using the XIMAGE detection algorithm and are in units of $10^{-2} \mathrm{cts} / \mathrm{s}$ in the band; fluxes are in units of $10^{-12}$ erg $\mathrm{cm}^{-2} \mathrm{~s}^{-1}$ in the $2-10 \mathrm{keV}$ band.

the QSO rest frame. Addition of this extra component provides a significant fit improvement (99.98\% confidence level using the $\mathrm{F}$ test), a steeper spectrum $(\Gamma=1.53 \pm 0.16)$ and a column density $\mathrm{N}_{H}=3 \pm 2 \times 10^{22}$ $\mathrm{cm}^{-2}$. Intrinsic absorption is a common property of radio loud/high redshift QSO (Page et al. 2005) and it is generally ascribed to the presence of absorbing material in the jet. However, this interpretation poses some difficulties as jets are expected to be efficient in removing gas in their vicinity. An alternative possibility is that the continuum is intrinsically curved (Tavecchio et al. 2007) and well described by a broken power law: indeed this model provides an equally significant improvement in the fit, a break energy at $1.55 \pm 0.15$ $\mathrm{keV}$ and a spectral flattening below $1-2 \mathrm{keV}$ of $\Delta \Gamma=0.5$ $\left(\Gamma_{1}=0.7 \pm 0.3\right.$ and $\left.\Gamma_{2}=1.2 \pm 0.4\right)$. Finally, a joint spectral fit to the Swift/XRT and INTEGRAL/IBIS data was also attempted. While we find a perfect match in spectral shape with $\Gamma=1.5 \pm 0.1$ (for an absorbed power law), the XRT data fall short of the INTEGRAL detection by a factor in the range 3-7 (much higher than the XRT/IBIS cross calibration constant which is typically around 1 , Masetti et al. 2007), implying some variability in the source flux between observations (see figure 3). Indeed, when the shorter XRT observation is also used ( see Table 1), a decrease in the source intensity becomes evident over the 6 day period of the Swift observational campaign. Variability in QSO is not unexpected and can be used to further characterized this new gamma-ray source. Assuming that the IBIS observation represents the average state of the source, we obtain rest frame luminosities ${ }^{6}$ of $0.3 \times 10^{48} \mathrm{erg} \mathrm{s}^{-1}$ in the X-ray $(2-10 \mathrm{keV})$ band, $2 \times$ $10^{48} \mathrm{erg} \mathrm{s}^{-1}$ at hard X-rays $(20-100 \mathrm{keV})$ and $5 \times 10^{48}$ erg $\mathrm{s}^{-1}$ in the soft gamma-ray (100-500 KeV) interval, i.e. MG3 J225155+2217 is an X/gamma-ray lighthouse shining from the edge of our Universe.

\section{WHAT TYPE OF QSO IS IGR J22517+2218/MG3 $\mathrm{J} 225155+2217$ ?}

Although a search in the NED database only shows 10 references, the sparse information available are sufficient to broadly characterize this new INTEGRAL source. The NVSS (NRAO VLA Sky Survey, Condon et al. 1998) image of MG3 J225155+2217 shows the source to be core dominated with no extended radio features and a $20 \mathrm{~cm}$ flux of 190 mJy. From the available data Kuhn (2004) estimated a radio loudness $\log R=3.9$ (well above the value used to define a source as radio loud) and a radio spectral index $\left(\mathrm{F}_{\nu} \propto \nu^{\alpha}\right)=-0.02$ (flat enough to classify the object as a FSRQ). Incidentally, the chance probability of finding such a bright radio source in the INTEGRAL error box is 0.01 , further supporting the association be-

${ }^{6}$ We adopt $\mathrm{H}_{o}=71 \mathrm{~km} \mathrm{~s}^{-1} \mathrm{Mpc}^{-1}, \Omega_{\Lambda}=0.73$ and $\Omega_{M}=0.27$

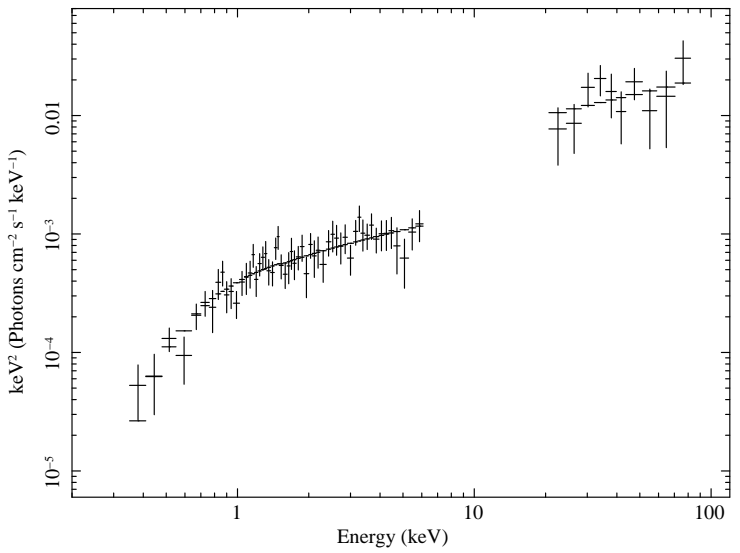

FIG. 3. - Broad band spectrum of IGR J22517+2218/MG3 J225155+2217 fitted with an intrinsically absorbed power law (continuous line in figure): stacked XRT data to the left and IBIS data to the right

tween IGR J22517+2218 and the QSO. Optically, MG3 $\mathrm{J} 225155+2217$ is a broad emission line AGN (FWHM $\geq 2000 \mathrm{~km} \mathrm{~s}^{-1}$ in the source rest frames, Falco et al. 1998); it is further labeled as a narrow absorption line (NAL) system due to the presence of absorption lines that are significantly narrower than the emission lines. These absorption features are seen in around 10\% of the spectra of high redshift QSO (Boksenberg 1978) and are either intrinsic to the source or the result of intervening gas between us and the QSO. Observations indicate that radio loud objects with flat radio spectra tend to avoid strong NAL, intrinsic or otherwise, and this make MG3 J225155+2217 peculiar in this respect (Ganguly et al. 2001). In X/gamma-rays, the source is bright with a hard X-ray spectral shape $(\Gamma=1.5)$; a deficit of soft photons in the spectrum can be either due to absorption local to the QSO or to intrinsic spectral curvature. The source is likely variable at high energies and possibly also at lower frequencies (Kuhn 2004).

Most of the above properties strongly suggest that MG3 $\mathrm{J} 225155+2217$ is a bright blazar, in which the emission is relativistically beamed and the SED double peaked. In figure 4, we construct the non-simultaneous rest frame SED of this enigmatic object by combining data from this work, from the HEASARC archive and from Kuhn (2004); to cover as many frequencies as possible we have also used upper limits obtained from the IRAS (Moshir et al. 1990) and EGRET (Hartmann et al. 1999) surveys. To deal with high energy variability, we have used in the SED the absorbed power law X/gamma-ray spectrum normalized to the IBIS flux. For comparison, we have also plotted in figure 4 the synchrotron inverse Compton model used to reproduced the SED of a blazar at comparable distance $([\mathrm{HB} 89] 0836+710, \mathrm{z}=2.17$, Tavecchio 
et al. 2000) scaled to match both the high energy data and the redshift of MG3 J225155+2217. The first striking feature of figure 4 is the extreme brightness of the source at X/gamma ray energies compared to the emission at lower frequencies: the $\mathrm{X}$-ray to radio flux ratio is $\sim 1000$ (or $\alpha_{x r}<0.75$ ), which makes it similar to high energy peaked blazars, i.e. those with the synchrotron peak in the X-ray band. The second important point is the shape of the infrared to optical continuum which is at odds with the location of the synchrotron peak at infrared frequencies as generally observed in FSRQ like [HB89] 0836+710 . Taken together, this indicates that this is a peculiar and rare object within the blazar population (Padovani 2007). Until recently, no high energy peaked FSRQ seemed to exist but some are now emerging such as the very powerful $\mathrm{z} \sim 4$ FSRQ ROXA J081009.9+384757.0 with a possible synchrotron peak in the X-ray band (Giommi et al. 2007). If the peak we see at around $100 \mathrm{keV}$ (or $\sim 400 \mathrm{keV}$ in the source rest frame) is due to the synchrotron component then : a) the blazar sequence in its simple formulation (Fossati et al. 1998) would be severely questioned by this result; b) the observed peak energy would be the highest ever measured in a FSRQ meaning that no difference in this parameter exists between low and high luminosity blazars; c) the Compton peak would be well into the gamma-ray band making the source a good target for AGILE and GLAST. Obviously, the sparse and non-simultaneous data coverage still leaves open the possibility of a more classical double humped interpretation of the source SED; but even in this case the object is strange as not only the above peculiarities have to be explained, but in this case the Compton peak would be at $\mathrm{MeV}$ energies, i.e. at lower energies than typically observed in FSRQ. Either way, MG3 J225155+2217 is quite atypical for its class and an extreme object in the blazar population; this makes it an interesting LABORATORY in where to test current blazars theories and so an object worth following up at all wavebands.

\section{CONCLUSIONS}

Through X-ray follow up observations with Swift/XRT, we have been able to identify the newly discovered INTEGRAL source, IGR J22517+2218 with MG3 J225155+2217, a QSO at $\mathrm{z}=3.668$. This makes IGR J22517+2218 the most distant object so far detected by INTEGRAL and the second most distant blazar ever observed above $20 \mathrm{keV}$. The source has many peculiar features which deserve more in depth studies: it is Aradio loud object with a flat spectrum; it hosts a NAL system in optical; it shows a spectral curvature in X-rays either intrinsic or due to high absorption in the source rest frame and it is variable at high energies. The source SED is also unusual among FSRQ as it is compatible with having the synchrotron peak in the $\mathrm{X} /$ gamma-ray band (i.e. much higher than generally observed) or alternatively with the Compton peak in the $\mathrm{MeV}$ range (i.e. lower than typically measured).

We acknowledge financial support from ASI (contracts $\mathrm{I} / \mathrm{R} / 008 / 07 / 0$ and $\mathrm{I} / 023 / 05 / 0$ ) and PPARC (grant $\mathrm{PP} / \mathrm{C} 000714 / 1)$. This research has made use of the NED NASA/IPAC Extragalactic Database (NED) operated by

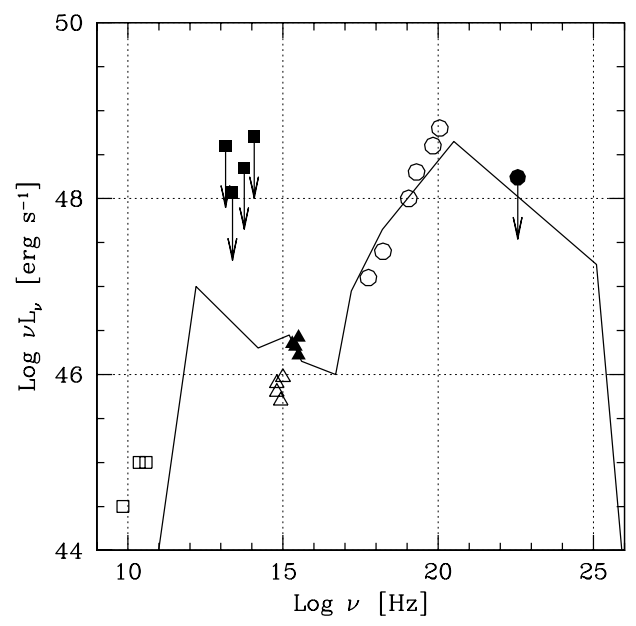

FIG. 4.- Non simultaneous rest frame SED of IGR $\mathrm{J} 22517+2218=$ MG3 J225155+2217. Data have been taken from HEASARC archive: radio (open squares),optical (full triangles); Moshir et al. (1990):far-infrared/IRAS (full squares); Kuhn (2004): near-infrared (open triangles); this work: X-rays/XRT-IBIS (opens circle) and Hartman et al. (1999):gamma-rays/EGRET (full circle). The line represents the SED of [HB89] 0836+710 normalized to MG3 J225155+2217 (see text)

JPL (Caltech) laboratory and of the HEASARC archive provided by NASA's Goddard Space Flight Center.

\section{REFERENCES}

Bird, A. J., Malizia, A., Bazzano, A., et al. 2007, Ap.J. Suppl., 170,175

Blom, J.J, Bennett,K., Bloemen, H., et al. 1996 A\&A Suppl. 120,507

Boksenberg A. 1978, Physika Scripta 17,205

Condon, J. J., Cotton, W. D., Greisen, E. W., et al. 1998, AJ, 115,1693

Donato, D., Sambruna, R.M., \& Gliozzi, M. 2005 A\&A 433, 1163

Dickey, J.M. \& Lockman, F.J. 1990, A.R.A.A. 28, 215

Falco, E.E.;Khochanek, C.S.; Munoz, J.A. 1998, Ap. J. 494, 47

Fossati, G., Maraschi, L., Celotti, A., Comastri, A., Ghisellini, 1998, MNRAS 299,433

Ganguly, R., Bond, N.A., Charlton J.C., et al. 2001, INAOE Electronic edition (http://www.inaoep.mx/ agn00/posters.html), 117

Gehrels, N., Chincarini, G., Giommi, P. et al. 2004, Ap.J. 611, 1005
Ghisellini, G., Celotti, A., Fossati, G., et al. 1998, MNRAS, 301, 451

Giommi,P.,Massaro,E.,Padovani, P., et al. 2007, A\&A 468,97

Hartman,R.C., Bertsch,D.L.,Bloom,S.D. et al., 1999 Ap.J. Suppl.123,79

Hill, J. E., Burrows, D. N., Nousek, J. A., et al. 2004, Proc. SPIE 5165,21

Krivonos, R., Revnivtsev, M., Lutovinov, A., et al. 2007, A\&A submitted (astroph 0701836)

Kuhn O.P. 2004, MNRAS 348, 647

Masetti, N., Landi, R., Pretorius, M.L. et al. 2007, A\&A 470, 331

Moshir, M., Copan, G., Conrow, T., et al. 1990, IRAS Faint Source Catalogue, version 2.0

Moretti, A., Campana, S. Tagliaferri, G., et al. 2004, Proc. SPIE 5165, 232

Page, K.L., Reeves, J.N., O'Brien, P.T., Turner, M.J.L. 2005 MNRAS 364, 195 
Padovani, P., 2007, Astrophys. Space Sci. in press

Sambruna, R. M., Tavecchio, F., Ghisellini, G. et al. 2007 Ap. J. in press (astroph. 07072925)

Tavecchio, F., Maraschi, L., Ghisellini, G., et al. 2000, Ap.J.543, 535

Tavecchio, F., Maraschi, M., Ghisellini G. et al. 2002 Ap.J. 575, 137
Tavecchio, F., Maraschi, L., Ghisellini, G., et al. 2007, Ap.J. in press 